\title{
Electrical Properties of Background-Limited Membrane-Isolation Transition-Edge Sensing Bolometers for Far-IR/Submillimeter Direct-Detection Spectroscopy
}

\author{
M. Kenyon · P.K. Day • C.M. Bradford · J.J. Bock • \\ H.G. Leduc
}

Received: 23 July 2007 / Accepted: 15 September 2007 / Published online: 19 January 2008

(C) Springer Science+Business Media, LLC 2008

\begin{abstract}
We built and measured the electrical properties of membrane-isolation transition-edge sensing bolometers (TESs) suitable for background-limited farIR/submillimeter direct-detection spectroscopy. Each TES consists of a Mo/Au bilayer patterned onto a suspended, thermally isolated absorber that is connected to the substrate through four $\mathrm{Si}_{x} \mathrm{~N}_{y}$ beams deposited by low pressure chemical vapor deposition (LPCVD). We fabricated TESs with straight and meander support beams. The dimensions of the meander (straight) support beams are $700 \mu \mathrm{m}(700 \mu \mathrm{m})$ long by $0.25 \mu \mathrm{m}(0.5 \mu \mathrm{m})$ thick by $0.35 \mu \mathrm{m}(0.5 \mu \mathrm{m})$ wide. We measured $I-V$ characteristics for these TESs and determined that the thermal conductance $G$ equals $72 \mathrm{fW} / \mathrm{K}$ (straight) and $19 \mathrm{fW} / \mathrm{K}$ (meander) for our best devices. The thermal conductance exhibits a $T^{1 / 2}$ dependence with temperature which is evidence of effective elastic scattering of the acoustic phonon modes. The transition temperatures $T_{c}$ for the same TESs are $T_{c}=137 \mathrm{mK}$ (straight) and $T_{c}=71 \mathrm{mK}$ (meander). If we assume the TESs are temperature-fluctuation noise limited, then the derived noise equivalent power $(\mathrm{NEP})$ equals $1.9 \times 10^{-19} \mathrm{~W} / \mathrm{Hz}^{1 / 2}$ (straight) and $6.1 \times 10^{-20} \mathrm{~W} / \mathrm{Hz}^{1 / 2}$ (meander), using our measured values for $G$ and $T_{c}$. The meander-beam TES has a derived NEP that is close to two orders of magnitude lower than the state-of-the-art. Finally, we measured an effective time constant $\tau$ of about $300 \mathrm{~ms}$ (straight) and $400 \mathrm{~ms}$ (meander) using electrical and optical pulses. These values for the NEP and $\tau$ for the meander-beam TES meet the requirements for the Background-Limited far-IR/Submillimeter Spectrograph (BLISS), a proposed NASA instrument.
\end{abstract}

Keywords Transition-edge sensor · Far-IR spectrometer ·

Submillimeter spectrometer $\cdot \mathrm{Si}_{3} \mathrm{~N}_{4}$ thermal transport

PACS $85.25 . \mathrm{Pb} \cdot 95.85 . \mathrm{Gn} \cdot 95.85 . \mathrm{Fm} \cdot 63.22 .+\mathrm{m}$

M. Kenyon $(\bowtie)$ P P.K. Day · C.M. Bradford · J.J. Bock · H.G. Leduc

Jet Propulsion Laboratory, Pasadena, CA 91109, USA

e-mail: matthew.e.kenyon@jpl.nasa.gov 


\section{Introduction}

Exploring the origins and the evolution of galaxies is of growing interest in the astrophysics community and requires the deployment of ambitious new farIR/submillimeter spectrometers into space. Moderate and high redshifted galaxies radiate in the far-IR/submillimeter $(40 \mu \mathrm{m}-600 \mu \mathrm{m})$ and these new spectrometers propose to measure the redshifts, the ionized and neutral gas fine structure lines, and the molecular lines of these galaxies.

One proposed mission currently receiving much attention is the Space Infrared Telescope for Cosmology and Astrophysics (SPICA), a Japanese observatory with a 3.5-meter telescope cooled to $4.5 \mathrm{~K}$ and deployed to L2 [10]. An instrument under study by NASA which could fly on SPICA is the Background-Limited Infrared/Submillimeter Spectrograph (BLISS) [1]. BLISS requires significant advances in state-of-the-art detector technology, however. In particular, the detectors on BLISS must be scalable to large arrays on the order of at least $10^{3}$ pixels, must be broadband over $40 \mu \mathrm{m}-600 \mu \mathrm{m}$, and must have a noise equivalent power (NEP) on the order of $10^{-20} \mathrm{~W} / \mathrm{Hz}^{1 / 2}$.

Membrane-isolation bolometers are a type of detector that are broadband and scalable to 1000's of pixels (i.e. SCUBA 2). To reach the NEP required for BLISS, one must reduce the fundamental intrinsic temperature-fluctuation noise in these bolometers. J.C. Mather derived an expression for this noise [9]:

$$
\mathrm{NEP}=\sqrt{\gamma 4 k_{\mathrm{b}} T^{2} G}
$$

where $k_{\mathrm{b}}$ is Boltzmann's constant, $T$ is the temperature of the thermistor, and $G$ is the thermal conductance between the substrate and the thermistor. The $\gamma$ term must be included to account for the temperature gradient along the support beam of the bolometer. If the thermal conductance $G$ is related to $T$ by the power law $G \propto T^{n}$, then $\gamma$ equals $1-(1+n / 2) t+(2+n)(2+3 n) t^{2} / 12+\cdots$ where $t=1-T_{S} / T\left(T_{S}\right.$ is the substrate temperature). Clearly from (1) one must reduce $G$ and $T$ in order to reduce the NEP. If we assume $T$ equals about $50 \mathrm{mK}$, one must lower $G$ to about $10 \mathrm{fW} / \mathrm{K}$ to obtain an NEP on the order of $10^{-20} \mathrm{~W} / \mathrm{Hz}^{1 / 2}$. We note that if $G \propto T^{1 / 2}$ (see Sect. 3 below), then NEP $\propto T^{5 / 4}$. A factor of 10 drop in $T$ causes the NEP to drop by about a factor of 20 .

In this paper, we discuss our progress in making membrane-isolation trans-itionedge sensing bolometers (TESs) which meet the rigorous demands of BLISS. Substantial investment in recent years in this detector technology and in a SQUIDbased multiplexer [2, 3, 5] readout has made this technology available for some large format applications, but the NEP of these detectors is still too high for their use in background-limited far-IR/submillimeter spectroscopy. Here we show that membrane-isolation TESs can reach the high sensitivities required by BLISS. In particular, we performed electrical measurements on membrane-isolation TESs and derive an NEP as low as $6 \times 10^{-20} \mathrm{~W} / \mathrm{Hz}^{1 / 2}$ from the values of $G$ and $T_{c}$ we measured for our best device. By increasing the aspect ratio of the support beams in the TES (see Results section), the NEP can be lowered even further. Finally, we measured the effective time constant $\tau$ of our membrane-isolation TESs and find that $\tau \simeq 300$ $400 \mathrm{~ms}$, making these detectors fast enough for BLISS. 


\section{Fabrication and Experimental Details}

Figure 1 shows micrographs of TESs which were fabricated at the Microdevice Laboratory (MDL) at JPL from bare $100 \mathrm{~mm} \mathrm{Si} \mathrm{wafers} \mathrm{to} \mathrm{finished} \mathrm{detectors.} \mathrm{The} \mathrm{ther-}$ mistors are composed of sputtered Mo/Au bilayer films that are lithographically patterned onto a perforated, rectangular absorber. Four support beams connect the absorber to the substrate. The entire suspended, thermally isolated support structure is composed of low-pressure chemical vapor deposition (LPCVD) $\mathrm{Si}_{x} \mathrm{~N}_{y}$. Sputtered niobium lines run over the support beams and electrically connect the thermistor to a dc circuit. Note the high aspect ratio of length-to-cross-sectional area of the support beams. This high ratio is necessary to lower the thermal conductance between the absorber and the substrate. The geometry of the $\mathrm{Si}_{x} \mathrm{~N}_{y}$ support beams for the TESs shown in Fig. 1(a) and (b) are straight and meandered, respectively. The straight beams are suitable for a $1 \mathrm{D}$ array while the meander beams can be packed into a 2D array.

We studied the electrical properties of these TESs using a ${ }^{3} \mathrm{He}-{ }^{4} \mathrm{He}$ dilution refrigerator at JPL. We mounted the devices inside a $\mathrm{Nb}$ box which was thermally anchored to the mixing chamber of the dilution refrigerator. We used a standard voltage biased circuit for our measurements [6]. First, the TES was connected in series to an inductor that couples the current through the TES to a SQUID. Second, a small shunt resistor $R_{S}$ was connected in parallel to the TES and inductor. A current source was used to supply a current to the circuit.

Since the thermal conductance for these TESs is designed to be on the order of $10 \mathrm{fW} / \mathrm{K}$, the bias power needed to bias the devices can be less than $1 \mathrm{fW}$ (see next section). To minimize extraneous power from the environment coupling into the bias lines of the TES and saturating the detector, we have filtered bias lines consisting of lossy coaxial cables running from room temperature to the mixing chamber of the dilution refrigerator and two low-pass filters mounted on the mixing chamber
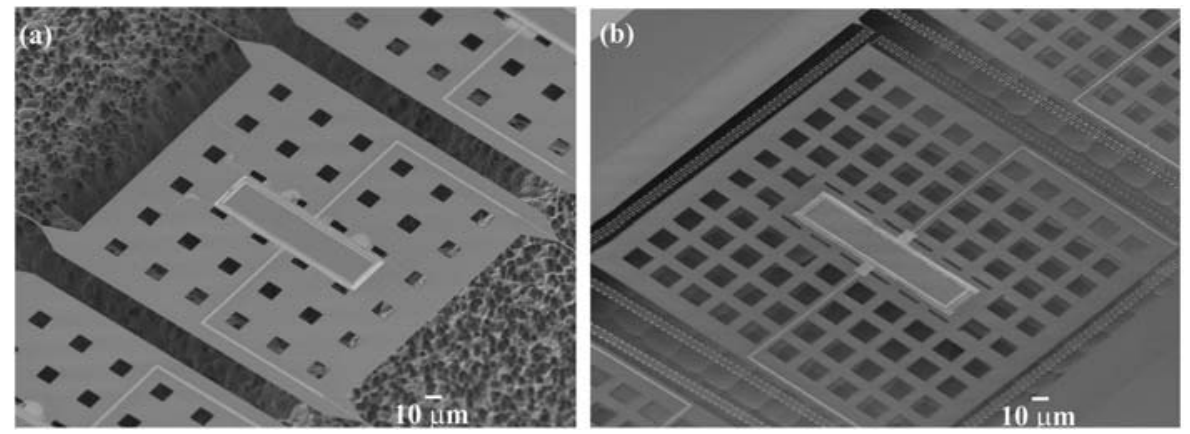

Fig. 1 (a) Bolometers with straight suspended $\mathrm{Si}_{x} \mathrm{~N}_{y}$ rsupport beams. The beam dimensions are $700 \mu \mathrm{m}$ long by $0.5 \mu \mathrm{m}$ thick by $0.5 \mu \mathrm{m}$ wide. (Note: the beams extend beyond the edge of the micrograph.) The thermistors are Mo/Au bilayer films deposited onto a perforated, rectangular $\mathrm{Si}_{x} \mathrm{~N}_{y}$ absorbers. The final release of the $\mathrm{Si}_{x} \mathrm{~N}_{y}$ structure is performed using a $\mathrm{XeF}_{2}$ etcher which causes the Si underneath the $\mathrm{Si}_{x} \mathrm{~N}_{y}$ support structure to appear pitted. (b) Bolometers with meander suspended $\mathrm{Si}_{x} \mathrm{~N}_{y}$ support beams. The beam dimensions are $700 \mu \mathrm{m}$ long by $0.25 \mu \mathrm{m}$ thick by $0.35 \mu \mathrm{m}$ wide. This pixel geometry is suitable for a close-packed 2D array 
with $3 \mathrm{~dB}$ rolloffs at $1.9 \mathrm{MHz}$ and $300 \mathrm{MHz}$, respectively. With the addition of a cold resistance divider, the dark power on these lines is low enough to measure the electrical properties of these TESs.

\section{Results and Discussion}

We measured the electrical properties of TESs like the ones shown in Fig. 1. (We note that the results of our measurements on TESs with other support beam geometries have been published elsewhere [7, 8]). Figure 2 shows $I-V$ curves for the TES with meander support beams [see Fig. 1(b)] for mixing chamber temperatures ranging from $24 \mathrm{mK}$ to $62 \mathrm{mK}$. The inset shows the TES bias power $P$ versus mixing chamber temperature $T$ for a fixed TES resistance $R=3 \mathrm{~m} \Omega$. From the inset, it is possible to determine the thermal conductance $G$, the transition temperature $T_{c}$ and, therefore, the NEP of the TES [see (1)].

The solid line in the inset of Fig. 2 is a power-law fit to the data. The transition temperature of the TES can be determined by using the heat flow equation:

$$
P=K\left(T_{c}^{n}-T_{m x}^{n}\right)
$$

where $P$ is the bias power through the TES, $K$ is a constant that depends on the geometry and material properties of the support beams, $T_{c}$ is the transition temperature of

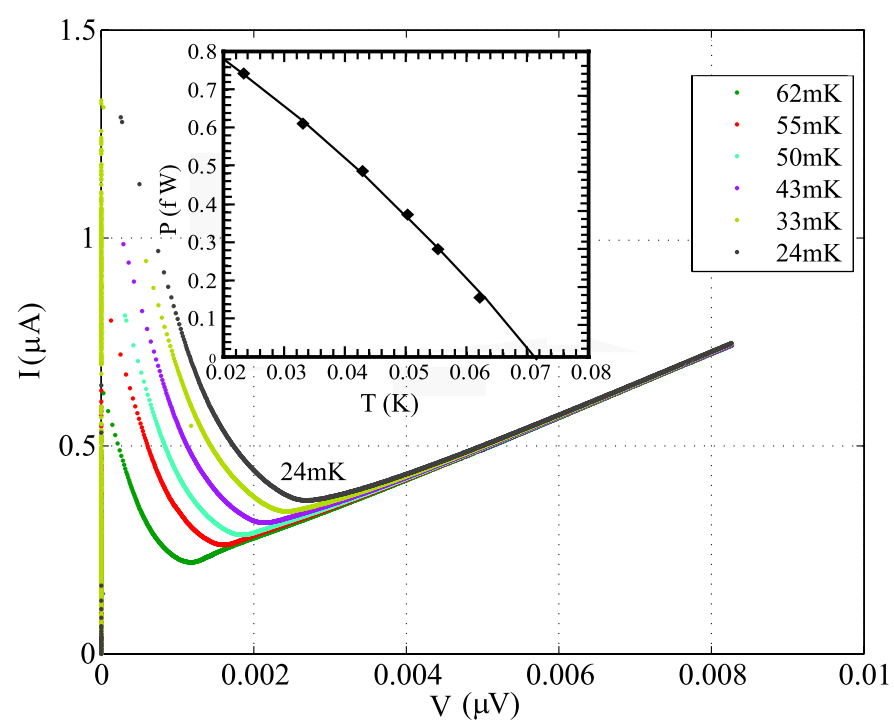

Fig. 2 (Color online) $I-V$ characteristic of TES for mixing chamber temperatures ranging from $24 \mathrm{mK}$ to $62 \mathrm{mK}$. Inset: Electrical bias power through the TES as a function of mixing chamber temperature. The resistance of the TES is set to $3 \mathrm{~m} \Omega$ for each temperature. The solid line is a power-law fit to the data with an exponent equal to 1.43 . A thermal conductance $G$ of $19 \mathrm{pW} / \mathrm{K}$ and a transition temperature of $T_{c}=71 \mathrm{mK}$ for the Mo/Au thermistor is determined by the slope and $x$-intercept of the line, respectively. From $G$ and $T_{c}$, one determines an NEP equal to $6 \times 10^{-20} \mathrm{~W} / \mathrm{Hz}^{1 / 2}$. Note that the bias power within the TES transition is less than $1 \mathrm{fW}$ 
the Mo/Au bilayer, and $T_{m x}$ is the temperature of the mixing chamber. Equation (2) suggests that as $P \rightarrow 0, T_{m x} \rightarrow T_{c}$. In other words, the $x$-intercept of solid line in the inset of Fig. 2 is the transition temperature of the Mo/Au bilayer and equals $71 \mathrm{mK}$. This value for $T_{c}$ agrees with 4-point resistance versus temperature measurements we performed on unsuspended Mo/Au bilayer films with the same nominal Mo-toAu ratio. Measurements of the $I-V$ curves for the TES with straight support beams [see Fig. 1(a)] reveal that the transition temperature of the Mo/Au bilayer is $137 \mathrm{mK}$.

The thermal conductance of the TES can be determined by the slope of the solid line $G \equiv d P / d T$ (see inset of Fig. 2) and equals $19 \mathrm{~W} / \mathrm{K}$. Interestingly, the thermal conductance $G$ depends on temperature $T$ according to $G \propto T^{0.43}$. Measurements on the straight-beam TES show that $G$ equals $72 \mathrm{fW} / \mathrm{K}$ and depends on temperature $T$ according to $G \propto T^{0.50}$. One might expect $G$ to depend linearly on $T$ because the width and thickness of the support beams are so small that the thermal transport is well inside the quantum regime. More specifically, the important modes for transmitting energy become the lowest acoustic modes of the $\mathrm{Si}_{x} \mathrm{~N}_{y}$ support beams. The higher energy modes freeze out as $T$ is lowered and only the so-called 1D modes contribute to the thermal conductance at low temperature [14]. If the thermal transport is ballistic, then it can be shown that the freezing out of higher energy modes causes the effective dimensionality of the beam to go from $3 \mathrm{D}$ to $1 \mathrm{D}$, resulting in the temperature behavior of $G$ crossing over from $G \sim T^{3}$ to $G=G_{Q}=\pi^{2} k_{b}^{2} T / 3 h \sim 1 \mathrm{pW} / \mathrm{K}^{2} \times T$. The quantity $G_{Q}$ is the universal quantum of thermal transport and is independent of the material properties of the support beam [14].

The fact that our measured $G$ from Fig. 2 is a factor of about four times lower than $G_{Q}$ and changes with $T$ according to $T^{1 / 2}$ implies that the thermal phonon transport along the support beams is not ballistic. Because the surfaces of the support beams have some surface roughness, transport phonons can scatter off the rough surfaces and lower the thermal conductance $G$. Surface scattering of transport phonons can also change the temperature dependent of $G$ [11-13]. B.A. Glavin [4] predicts that the thermal conductance of support beams when effective elastic scattering is present will follow a $T^{1 / 2}$ dependence. To our knowledge, this result is the first confirmation of Glavin's theory of heat transfer in suspended beams.

We note that the thermal conductance $G$ we measured for the straight- and meander-beam TESs appear to scale proportionately with cross-sectional area. If we scale the cross-sectional area of the straight-beam TES to match the meander-beam cross-sectional area and use (2) to scale the straight-beam $T_{c}$ from $137 \mathrm{mK}$ to $71 \mathrm{mK}$, then $G$ for the straight-beam TES drops from $72 \mathrm{fW} / \mathrm{K}$ to $19 \mathrm{fW} / \mathrm{K}$, agreeing with the value we measured for the meander-beam TES.

Finally, we measured the effective time constant $\tau$ of the meander-beam TES as a function of the TES bias voltage $V_{b}$ (see Fig. 3). We performed the measurement by coupling optical pulses from an LED through an optical fiber into the sample box of the TES. Since the TES is voltage biased, there is negative electrothermal feedback [6] and the effective time constant is $\tau=C / G(1+P \alpha / G T)$ where $C$ is the heat capacity, $G$ is the thermal conductance, $P$ is the bias power, and $\alpha$ is $(T / R) \times(d R / d T)$ of the bolometer. Using (2) and the fact that $G=n K$, the effective time constant simply becomes $\tau=C / G(1+\alpha / n)$. Figure 3 shows that $\tau$ reaches a minimum value of about $0.37 \mathrm{~s}$ when $V_{b}=2.7 \mathrm{~V}$. If we assume $\alpha=10-20$, then 
Fig. 3 (Color online) The time response of the TES for eight different bias voltage $V_{b}$ values. LED pulses $(\lambda=660 \mathrm{~nm})$ were coupled into the device through a fiber optic line fed into sample box of the TES. Inset shows the effective time constant $\tau$ for different bias voltages $V_{b}$. The electrothermal feedback is a maximum at $V_{b}=2.7 \mathrm{~V}$

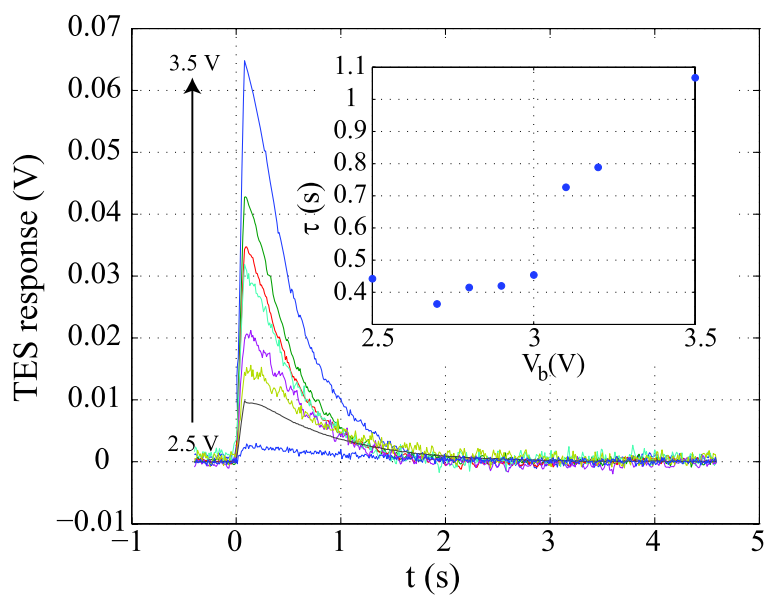

the heat capacity $C$ of the TES is estimated to be between $150-300 \mathrm{fJ} / \mathrm{K}$. We suspect the heat capacity is dominated by defect states in the $\operatorname{Si}_{x} \mathrm{~N}_{y}$ absorber. The time response for the TES shown in Fig. 1(a) is slightly faster. We measured an effective time constant equal to $300 \mathrm{~ms}$.

\section{Conclusions}

We have shown that membrane-isolation TESs are suitable for the next generation of proposed background-limited far-IR/submillimeter spectrometers. These TESs have a derived NEP that is close to two orders of magnitude lower than the current stateof-the-art. Moreover, these ultra-low NEP bolometers have an effective time constant well below $1 \mathrm{~s}$, making them fast enough for the next generation spectrometers. Future plans include measuring the electrical NEP directly and understanding how microphonics effect the performance of these ultra-low NEP TESs.

Acknowledgements This research was carried out at the Jet Propulsion Laboratory and Caltech under contract with the National Aeronautics and Space Administration.

\section{References}

1. C.M. Bradford et al., Proc. SPIE 4850, 1137 (2003)

2. J.A. Chervenak et al., Appl. Phys. Lett. 74, 4043 (1999)

3. W.B. Doriese et al., Nucl. Instrum. Methods A 520, 559 (2003)

4. B.A. Glavin, Phys. Rev. Lett. 86, 4318 (2001)

5. W.S. Holland et al., Proc. Soc. Photo. Opt. Instrum. Eng. 4855, 479 (2003)

6. K.D. Irwin, Appl. Phys. Lett. 66, 1998 (1995)

7. M. Kenyon et al., Nucl. Instrum. Methods A 559, 456 (2006)

8. M. Kenyon et al., Proc. SPIE 6275, 627508 (2006) 
9. J.C. Mather, Appl. Opt. 21, 1125 (1982)

10. T. Matsumoto, Proc. SPIE 5487, 1501 (2004)

11. G. Palasantzas, Phys. Rev. B 70, 153404 (2004)

12. D.H. Santamore, M.C. Cross, Phys. Rev. B 62, 184306 (2001)

13. D.H. Santamore, M.C. Cross, Phys. Rev. B 66, 144302 (2002)

14. K. Schwab et al., Nature 404, 974 (2000) 